\title{
Statistical Mechanics and Lorentz Violation
}

\author{
Don Colladay* and Patrick McDonald ${ }^{\dagger}$ \\ New College of Florida
}

(Dated: July 29, 2021)

\begin{abstract}
The theory of statistical mechanics is studied in the presence of Lorentz-violating background fields. The analysis is performed using the Standard-Model Extension (SME) together with a Jaynesian formulation of statistical inference. Conventional laws of thermodynamics are obtained in the presence of a perturbed hamiltonian that contains the Lorentz violating terms. As an example, properties of the nonrelativistic ideal gas are calculated in detail. To lowest order in Lorentz violation, the scalar thermodynamic variables are only corrected by a rotationally invariant combination of parameters that mimics a (frame dependent) effective mass. Spin couplings can induce a temperature independent polarization in the classical gas that is not present in the conventional case. Precision measurements in the residual expectation values of the magnetic moment of Fermi gases in the limit of high temperature may provide interesting limits on these parameters.
\end{abstract}

\section{INTRODUCTION}

The notion that the minimal standard model serves as a low energy limit to a more fundamental theory which includes a quantum description of gravity has led to the development of theories which extend the standard model and predict qualitatively different physical phenomena [1]. In this context, a framework for studying the effects of spontaneous Lorentz symmetry breaking and possible resulting CPT violation within the context of conventional quantum field theory has been developed 2, 3] and studied intensively [4]. This framework formally contains all possible operators utilizing standard model fields that satisfy coordinate reparametrization invariance and is called the Standard-Model Extension (SME). The minimal version of the SME is restricted to exhibit a number of useful properties: it preserves energy-momentum conservation, observer Lorentz invariance, conventional quantization, hermiticity, microcausality, positivity of the energy, gauge invariance and power counting renormalizability. In addition, the framework provides a generic model for any theory which extends the standard model and provides for spontaneous Lorentz symmetry breaking and CPT violation. For example, Lorentz violation may arise in noncommutative field theory [5] or random dynamics models $[\underline{6}$.

To date, there has been no confirmed evidence for Lorentz violation, hence it is reasonable to assume that any violation must be small in conventional laboratory frames (concordant frames) 7]. Various experiments utilizing mesons [8, 9, 10], baryons 11, 12, 13], electrons [14, 15, 16], photons [17, 18, 19, 20, 21], and muons [22] have reached a precision that probes these parameters at Planck-suppressed scales. Recent analysis have also been extended to the neutrino sector [23, 24], instantons [25], supersymmetric models [26], and the gravitational sector [27].

* colladay@ncf.edu

tmcdonald@ncf.edu
Statistical mechanics has been applied to rotationally invariant CPT-violating terms in the SME to provide a mechanism for baryogenesis in thermal equilibrium [28], but a detailed exposition of the formalism for general Lorentz violation is lacking. It is the goal of this paper to address this issue.

A general framework is provided in this paper for performing statistical mechanics calculations within the context of the SME. The required assumptions regarding statistical inference and the definitions of relevant thermodynamic quantities are given. A complete analysis of the effects of all Lorentz-violating terms on a nonrelativistic ideal gas is performed. The corresponding thermodynamics is studied and the various changes in thermodynamic quantities are analyzed. In section II, the notation and the relevant formalism for performing statistical mechanics calculations within the context of the SME is presented. Section III contains calculations of the relevant thermodynamic quantities for single particle systems. Section IV generalizes the result to a classical gas with a variable particle number and incorporates the chemical potential. Section $\mathrm{V}$ generalizes the result to a quantum gas of fermions and section VI gives corresponding results for spin-0 bosons. The conclusions are gathered in section VII.

\section{NOTATION AND FORMALISM}

Let $\left\{\psi_{i}\right\}_{i=1}^{\infty}$ be a collection of states and suppose that $\left\{f_{j}\right\}_{j=1}^{l}$ is a finite collection of real valued functions on the collection of states. Given a distribution of states, $q_{i}=q\left(\psi_{i}\right)$, we denote by brackets the corresponding expectations; $\left\langle f_{j}\right\rangle=\sum_{i} f_{j}\left(\psi_{i}\right) q_{i}$. We denote the (information) entropy associated to the distribution $q_{i}$ by

$$
S=-k \sum_{i} q_{i} \ln \left(q_{i}\right)
$$

where $k$ is a positive constant that will later be identified with Boltzmann's constant.

Suppose we are given an observation of the mean values $\left\{\left\langle f_{j}\right\rangle\right\}_{j=1}^{l}$ and we seek a distribution that best fits 
our observation. In his approach to statistical inference, Jaynes 29] argues that one should choose a distribution which maximizes (11) subject to the constraints on expectations given by the observations. In so doing, a now standard argument via variational calculus leads immediately to the solution

$$
q_{i}=\frac{e^{-\sum_{j=1}^{l} \lambda_{j} f_{j}\left(\psi_{i}\right)}}{Z},
$$

where the Lagrange multipliers, $\lambda_{j}$, are real constants and $Z$ is the partition function, $Z(\lambda)=$ $\sum_{i} e^{-\sum_{j=1}^{l} \lambda_{j} f_{j}\left(\psi_{i}\right)}$. From (2) it follows that

$$
\left\langle f_{j}\right\rangle=-\frac{\partial}{\partial \lambda_{j}} \ln (Z)
$$

If, in addition to being functions of state, the $f_{j}$ are permitted to depend on a finite collection of parameters, $a_{j}, 1 \leq j \leq m$, then it is easy to see that

$$
\sum_{j} \lambda_{j}\left\langle\frac{\partial f_{j}}{\partial a_{k}}\right\rangle=-\frac{\partial}{\partial a_{k}} \ln (Z) .
$$

The resulting maximal entropy is given by

$$
S_{\max }=k \ln Z+k \sum_{j} \lambda_{j}\left\langle f_{j}\right\rangle .
$$

Variation of the maximal entropy with respect to the parameters $\lambda_{j}, \alpha_{j}$, and $\left\langle f_{j}\right\rangle$ yields the relation

$$
d S=\sum_{k} \frac{\partial \ln Z}{\partial \alpha_{k}} d \alpha_{k}+\sum_{j} \lambda_{j} d\left\langle f_{j}\right\rangle .
$$

As is clear from our development, (2)-(3) are formal rules of inference which follow for any system described as above, under the assumption of maximum entropy. Jaynes applies these formal rules to the study of equilibrium thermodynamics. More precisely, suppose that we consider a system of identical particles constrained to lie in a box of volume $V$. Suppose that $f_{1}=E$ denotes the energy levels corresponding to the possible states of the system and that $f_{2}=N$ denotes the number of particles in the system. Given observations of the mean values, $\langle E\rangle,\langle N\rangle$, one obtains the partition function for the grand canonical ensemble:

$$
Z(\alpha, \beta)=\sum_{i, j} e^{-\beta E_{j}-\alpha N_{i}},
$$

where we have identified the Lagrange multiplier $\lambda_{1}$ with the scaled inverse temperature $\beta=\frac{1}{k T}$ and the Lagrange multiplier $\lambda_{2}=\alpha$ with $-\beta \mu$, where $\mu$ is the chemical potential.

Allowing the energy to depend on the volume $V$ and setting $a_{1}=V$ in (4) we get the usual description of pressure

$$
P=-\left\langle\frac{\partial E}{\partial V}\right\rangle=\frac{1}{\beta} \frac{\partial}{\partial V} \ln (Z) .
$$

Equations (11)-(8) give the central features of thermodynamics including expressions for the entropy as a function of temperature, volume and particle number, and the usual first law of thermodynamics. The standard expression for average energy and particle number are given by (31) and the expressions for specific heat at constant volume and at constant pressure follow by taking the appropriate derivatives of the entropy:

$$
\begin{aligned}
& C_{V}=T\left(\frac{\partial S}{\partial T}\right)_{\langle N\rangle, V}=\left(\frac{\partial\langle E\rangle}{\partial T}\right)_{\langle N\rangle, V}, \\
& C_{P}=T\left(\frac{\partial S}{\partial T}\right)_{\langle N\rangle, P} .
\end{aligned}
$$

Thermodynamic potentials such as the Helmholtz free energy are defined in the usual way.

As was emphasized by Jaynes, the method of maximum entropy provides accurate predictions of thermodynamic properties, assuming empirically accurate mean values and laws of motion embodied in the associated hamiltonian. For the purpose of probing the thermodynamics of systems of particles with symmetry violations, the method offers a simple framework for generating results. All that remains is to specify the relevant underlying hamiltonian.

In the case of free spin- $\frac{1}{2}$ Dirac fermions $\psi$ of mass $m$, the SME is determined by the lagrangian [2]

$$
\mathcal{L}=\frac{1}{2} i \bar{\psi} \Gamma_{\nu} \stackrel{\leftrightarrow}{\partial^{\nu}} \psi-\bar{\psi} M \psi
$$

where

$$
\begin{aligned}
\Gamma_{\nu} & =\gamma_{\nu}+c_{\mu \nu} \gamma^{\mu}+d_{\mu \nu} \gamma_{5} \gamma^{\mu}+e_{\nu}+i f_{\nu} \gamma_{5}+\frac{1}{2} g_{\lambda \mu \nu} \sigma^{\lambda \mu}, \\
M & =m+a_{\mu} \gamma^{\mu}+b_{\mu} \gamma_{5} \gamma^{\mu}+\frac{1}{2} H_{\mu \nu} \sigma^{\mu \nu} .
\end{aligned}
$$

In the above expressions $a_{\mu}, b_{\mu}, c_{\mu \nu}, d_{\mu \nu}, e_{\mu}, f_{\mu}, g_{\lambda \mu \nu}$ and $H_{\mu \nu}$ are real fixed background parameters which determine the Lorentz violation. For low energy applications the associated nonrelativistic hamiltonian $H$ has been obtained using a generalized Foldy-Wouthuysen transformation 30]. To second order in $\mathbf{p} / m$, this hamiltonian is given by 31 .

$$
H=\frac{p^{2}}{2 m}+H_{L V}
$$

with

$$
\begin{aligned}
H_{L V}= & A+B_{j} \sigma^{j}+C_{j} \frac{p_{j}}{m}+D_{j k} \frac{p_{j}}{m} \sigma^{k}+ \\
& F_{j k} \frac{p_{j} p_{k}}{2 m}+G_{j k l} \frac{p_{j} p_{k}}{2 m} \sigma^{l},
\end{aligned}
$$

where common terms in the original lagrangian (11) are collected:

$$
\begin{gathered}
A=\left(a_{0}-m c_{00}-m e_{0}\right) \\
B_{j}=\left(-b_{j}+m d_{j 0}-\frac{1}{2} m \epsilon_{j k l} g_{k l 0}+\frac{1}{2} \epsilon_{j k l} H_{k l}\right),
\end{gathered}
$$




$$
\begin{gathered}
C_{j}=\left[a_{j}-m\left(c_{0 j}+c_{j 0}\right)-m e_{j}\right] \\
D_{j k}=\begin{array}{c}
{\left[-b_{0} \delta_{j k}+m\left(d_{k j}+d_{00} \delta_{j k}\right)\right.} \\
\left.+m \epsilon_{k l m}\left(\frac{1}{2} g_{m l j}+g_{m 00} \delta_{j l}\right)+\epsilon_{j k l} H_{l 0}\right],(17) \\
F_{j k}=-2\left[\left(c_{j k}+\frac{1}{2} c_{00} \delta_{j k}\right)\right] \\
G_{j k l}=2\left\{\left[\left(d_{0 j}+d_{j 0}\right)\right.\right. \\
\left.-\frac{1}{2}\left(b_{j} / m+d_{j 0}+\frac{1}{2} \epsilon_{j m n}\left(g_{m n 0}+H_{m n} / m\right)\right)\right] \delta_{k l} \\
+\frac{1}{2}\left(b_{l} / m+\frac{1}{2} \epsilon_{l m n} g_{m n 0}\right) \delta_{j k} \\
\left.-\epsilon_{j l m}\left(g_{m 0 k}+g_{m k 0}\right)\right\}
\end{array}
\end{gathered}
$$

The hamiltonian is invariant under spatial translations, therefore the logarithm of the associated grand partition function scales with volume as in the conventional case. Using Eq. (8) together with this property yields the relation

$$
\frac{P V}{k T}=\ln (Z)
$$

\section{SINGLE PARTICLE SYSTEMS}

We first consider a system consisting of a single free spin- $\frac{1}{2}$ particle governed by the hamiltonian $H$ appearing in (12), constrained to a cube of side length $L$. For small violations of Lorentz symmetry, we derive the corresponding perturbations to the statistical mechanics using the single particle partition function. This simplifies the initial analysis by eliminating the need to discuss the chemical potential.

The standard unperturbed solutions are written in the form

$$
\psi_{\mathbf{n}, s}^{(0)}(\mathbf{x})=\prod_{i} \sin \left(\frac{n_{i} \pi x_{i}}{L}\right) \chi_{s}
$$

where $\mathbf{n}=\left(n_{1}, n_{2}, n_{3}\right)$ is a triple of positive integers and $s \in\{1,-1\}$ denotes a sign. Note that the two-component spinor $\chi_{s}$ may depend on $\mathbf{n}$. The corresponding unperturbed energy levels are written as

$$
E_{\mathbf{n}, s}^{(0)}=\frac{\pi^{2} \hbar^{2}}{2 m L^{2}} n^{2}
$$

The first order correction to the energy levels due to the Lorentz-violating terms are found using standard degenerate perturbation theory as:

$$
\left\langle\psi_{\mathbf{n}, s}\left|H_{L V}\right| \psi_{\mathbf{n}, s}\right\rangle=\frac{\pi^{2} \hbar^{2}}{2 m L^{2}}\left(A n^{2}+\sum_{i} F_{i i} n_{i}^{2}+s|\mathcal{G}(\mathbf{n})|\right),
$$

where the vector $\mathcal{G}(\mathbf{n})$ is defined with components

$$
(\mathcal{G}(\mathbf{n}))_{j} \equiv \frac{2 m L^{2}}{\pi^{2} \hbar^{2}} B_{j}+\sum_{i} G_{i i j} n_{i}^{2}
$$

The $\mathbf{n}$ dependent spinors $\chi_{s}$ have been chosen to satisfy $\mathcal{G}(\mathbf{n}) \cdot \sigma \chi_{s}=s \chi_{s}$ and therefore diagonalize $H_{L V}$. Note that $C$ and $D$ perturbations depend linearly on momentum and therefore don't contribute a correction to the energies.

The perturbed energy is written as

$$
E_{\mathbf{n}, s}=E_{\mathbf{n}, s}^{(0)}+\delta E_{\mathbf{n}, s},
$$

with $\delta E_{\mathbf{n}, s}$ given by the matrix elements (23). The partition function for a single particle becomes

$$
Z^{(1)}=\sum_{\mathbf{n}} e^{-\beta E_{\mathbf{n},+}}+e^{-\beta E_{\mathbf{n},-}}
$$

Approximating the sum on the right hand side of (26) by the appropriate integrals, we obtain expressions for partition functions corresponding to hamiltonians with Lorentz-violating terms. The partition function is even in the spin-dependent correction terms and therefore there are no lowest order corrections contributed by $B$ or $G$. The $A$ and $F$ terms correct the partition function as

$$
\begin{aligned}
Z^{(1)} & \simeq 2 e^{-\beta A} \frac{4 \pi}{8} \int_{0}^{\infty} n^{2} e^{-\beta \frac{\pi^{2} \hbar^{2}}{2 m L^{2}}\left(n^{2}+\sum F_{i i} n_{i}^{2}\right)} d n \\
& \simeq 2 e^{-\beta A} n_{Q} V\left(1-\frac{1}{2} \operatorname{Tr}(F)\right)
\end{aligned}
$$

where $V$ is the volume of the box, $n_{Q}=\left(m / 2 \beta \pi \hbar^{2}\right)^{3 / 2}$ is the quantum concentration, and $\operatorname{Tr}(F)=\sum F_{i i}$.

Using the relationship $\left\langle E^{(1)}\right\rangle=-(\partial / \partial \beta) \ln \left(Z^{(1)}\right)$ (cf (3)), it follows from (27) that only the $A$ term corrects the energy. The $A$ term corresponds to a constant shift in all of the energy levels, hence it is possible to redefine the zero point of the energy 32] to eliminate this contribution.

The correction to the partition function due to the $F$ term can be incorporated into an effective mass for the fermion

$$
m^{*}=\left(1-\frac{1}{3} \operatorname{Tr}(F)\right) m,
$$

because only the rotationally invariant trace component contributes. The $\operatorname{Tr}(F)$ term appears to be a trivial scaling that can be absorbed into the mass, but this is not quite true because the effective mass violates boost invariance. This means that the effective mass can be different in various laboratory frames. However, such effects will be relativistically suppressed and therefore difficult to observe using Earth-based experiments.

More interesting is the correction to the expectation value of the spin. In this case, only the $B$ and $G$ terms will contribute. The expectation is calculated as

$$
\begin{aligned}
\left\langle\mathbf{s}^{(1)}\right\rangle & =\left(Z^{(1)}\right)^{-1} \sum_{\mathbf{n}, s} \frac{s \mathcal{G}(\mathbf{n})}{|\mathcal{G}(\mathbf{n})|} e^{-\beta \frac{\pi^{2} \hbar^{2}}{2 m L^{2}}\left(n^{2}+s|\mathcal{G}(\mathbf{n})|\right)} \\
& \simeq-\left(n_{Q} V\right)^{-1} \beta \frac{\pi^{2} \hbar^{2}}{2 m L^{2}} \sum_{\mathbf{n}} \mathcal{G}(\mathbf{n}) e^{-\beta \frac{\pi^{2} \hbar^{2}}{2 m L^{2}} n^{2}} \\
& \simeq-\beta \mathbf{B}-\frac{1}{2} \operatorname{Tr}(\mathbf{G}),
\end{aligned}
$$


where the vector $(\operatorname{Tr}(\mathbf{G}))_{k} \equiv \sum_{i} G_{i i k}$ is defined. Note that while it is not in general possible to simultaneously diagonalize the contribution from $B$ and $G$ terms, the same computation allows us to treat the case where both terms occur. The corresponding spin expectations decouple.

\section{CLASSICAL GAS}

We now introduce the chemical potential and consider a classical gas of free spin- $\frac{1}{2}$ Dirac Fermions. As in section II, $N$ denotes the (variable) particle number for the system. The grand partition function for the system can be written in terms of the single-particle partition function as

$$
Z^{(C)}=\exp \left(e^{-\alpha^{(C)}} Z^{(1)}(\beta)\right),
$$

where $\alpha^{(C)}=-\beta \mu^{(C)}$ and $\mu^{(C)}$ is the chemical potential of the classical gas. Thus equation (27) also gives the first order corrections for the grand partition function. Using the grand partition function, the resulting expressions for expected particle number and energy are

$$
\begin{aligned}
\left\langle N^{(C)}\right\rangle & =e^{-\alpha} Z^{(1)}, \\
\left\langle E^{(C)}\right\rangle & =\frac{3}{2}\left\langle N^{(C)}\right\rangle k T .
\end{aligned}
$$

Since $P V=k T \ln Z^{(C)}$, it follows that there is no change in the classical ideal gas law, even in the presence of Lorentz violation.

Solving for the chemical potential $\mu^{(C)}$ (to lowest order in Lorentz violating parameters) yields

$$
\mu^{(C)}=-k T\left(\ln \left(\frac{2 n_{Q}}{n^{(C)}}\right)-\frac{1}{2} \operatorname{Tr}(F)\right),
$$

where $n_{Q}$ is the quantum concentration and $n^{(C)} \equiv$ $\left\langle N^{(C)}\right\rangle / V$ is the concentration of the classical gas. We conclude that in the presence of Lorentz violation there is a change in the chemical potential as expected from the effective mass argument presented in the previous section.

The corresponding entropy $S^{(C)}$ is found using equation (5) as

$$
S^{(C)}=\alpha^{(C)}\left\langle N^{(C)}\right\rangle k+\frac{5}{2}\left\langle N^{(C)}\right\rangle k .
$$

Using (33), the modified Sackur-Tetrode equation is

$$
S^{(C)}=\left\langle N^{(C)}\right\rangle k\left[\frac{5}{2}-\frac{1}{2} \operatorname{Tr}(F)+\ln \left(\frac{2 n_{Q}}{n^{(C)}}\right)\right] \text {. }
$$

From (35) and (9)-(10) it is clear that there is no change to the specific heat.

Finally, we verify that the expectation of the spin is in fact the single particle result in equation (29) times the expected particle number

$$
\left\langle\mathbf{s}^{(C)}\right\rangle=-\left\langle N^{(C)}\right\rangle\left[\beta \mathbf{B}+\frac{1}{2} \operatorname{Tr}(\mathbf{G})\right] .
$$

\section{QUANTUM GAS - FERMIONS}

Next, the quantum occupancy of the orbitals is restricted to be 0 or 1 and the low-temperature limit is analyzed. With notation from previous sections, the partition function for the grand canonical ensemble associated to a Fermi gas is

$$
Z^{(Q)}(\alpha)=\prod_{\mathbf{n}, s}\left(1+e^{-\alpha} e^{-\beta E_{\mathbf{n}, s}}\right)
$$

In the unperturbed case, the grand partition function is calculated using the appropriate integral approximation as (zero subscripts represent unperturbed quantities)

$$
\begin{aligned}
\ln \left(Z_{0}^{(Q)}\left(\alpha_{0}\right)\right) & =\pi \int_{0}^{\infty} n^{2} \ln \left(1+e^{-\alpha_{0}-\beta \frac{\pi^{2} \hbar^{2}}{2 m L^{2}} n^{2}}\right) d n \\
& =\frac{2}{\lambda^{3}} V f_{\frac{5}{2}}\left(e^{-\alpha_{0}}\right),
\end{aligned}
$$

where $\lambda=h /(2 \pi m k T)^{\frac{1}{2}}$ is the thermal wavelength (related to the quantum concentration by $\left.1 / \lambda^{3}=n_{Q}\right)$ and $f_{\nu}\left(e^{-\alpha}\right)$ is the Fermi-Dirac integral

$$
f_{\nu}\left(e^{-\alpha}\right)=\frac{1}{\Gamma(\nu)} \int_{0}^{\infty} \frac{x^{\nu-1}}{e^{\alpha} e^{x}+1} d x
$$

Using (3) gives the standard results

$$
\begin{aligned}
\left\langle N_{0}^{(Q)}\left(\alpha_{0}\right)\right\rangle & =\frac{2}{\lambda^{3}} V f_{\frac{3}{2}}\left(e^{-\alpha_{0}}\right), \\
\left\langle E_{0}^{(Q)}\left(\alpha_{0}\right)\right\rangle & =\frac{3}{2}\left\langle N_{0}^{(Q)}\left(\alpha_{0}\right)\right\rangle k T \frac{f_{\frac{5}{2}}\left(e^{-\alpha_{0}}\right)}{f_{\frac{3}{2}}\left(e^{-\alpha_{0}}\right)},
\end{aligned}
$$

and the quantum ideal gas law

$$
\frac{P}{n k T}=\frac{f_{\frac{5}{2}}\left(e^{-\alpha_{0}}\right)}{f_{\frac{3}{2}}\left(e^{-\alpha_{0}}\right)}
$$

As in the classical case, there are no first order corrections to the partition function for Lorentz violating terms given by the coefficients $B, C, D$ and $G$ appearing in (13). For violating terms of type $F$, a change of variables in the integral (38) gives first order corrections for the partition function which we write as

$$
\ln \left(Z^{(Q)}(\alpha)\right) \simeq\left(1-\frac{1}{2} \operatorname{Tr}(F)\right) \ln \left(Z_{0}^{(Q)}(\alpha)\right)
$$

As in the classical case, only the rotationally invariant component of $F$ contributes, therefore it is possible to absorb the term into an effective mass as before. Standard calculations then immediately give the results

$$
\begin{aligned}
\left\langle N^{(Q)}(\alpha)\right\rangle & =\left(1-\frac{1}{2} \operatorname{Tr}(F)\right) \frac{2}{\lambda^{3}} f_{\frac{3}{2}}\left(e^{-\alpha}\right), \\
\left\langle E^{(Q)}(\alpha)\right\rangle & =\frac{3}{2}\left\langle N^{(Q)}(\alpha)\right\rangle k T \frac{f_{\frac{5}{2}}\left(e^{-\alpha}\right)}{f_{\frac{3}{2}}\left(e^{-\alpha}\right)},
\end{aligned}
$$


as well as the ideal gas law

$$
\frac{P}{n^{(Q)} k T}=\frac{f_{\frac{5}{2}}\left(e^{-\alpha}\right)}{f_{\frac{3}{2}}\left(e^{-\alpha}\right)}
$$

where $n^{(Q)}$ is the concentration of the quantum gas (not to be confused with the quantum concentration $n_{Q}$ defined earlier). Note that the ideal gas law is in fact modified in the quantum case due to its dependence on $\alpha \neq \alpha_{0}$. The correction due to $F$ may be incorporated easily by replacing $\lambda(m)$ by $\lambda\left(m^{*}\right)$ using the effective mass given in equation (28).

From (39) it is clear that the map $\alpha \rightarrow f_{\frac{3}{2}}\left(e^{-\alpha}\right)$ is strictly monotonic and thus invertible. Writing the inverse as $\mathcal{F}$, we have a formal expression for the chemical potential

$$
\mu^{(Q)} \simeq-k T \mathcal{F}\left(\frac{\lambda^{3}\left(m^{*}\right) n^{(Q)}}{2}\right)
$$

Equation (47) demonstrates that conventional formulas can be used to obtain the relevant quantities. These formulas can be found in a standard statistical mechanics text such as [33]. For instance, the chemical potential at zero temperature defines the Fermi energy and is modified as

$$
\mu^{(Q)}(T=0) \equiv \mathcal{E}_{\mathcal{F}} \simeq \mathcal{E}_{\mathcal{F}}{ }^{(0)}\left(1+\frac{1}{3} \operatorname{Tr}(F)\right)
$$

where $\mathcal{E}_{\mathcal{F}}{ }^{(0)}=\left(\hbar^{2} / 2 m\right)\left(3 \pi^{2} n^{(Q)}\right)^{2 / 3}$ is the conventional Fermi energy. Approximating to the next highest order in temperature gives

$$
\mu^{(Q)} \simeq \mathcal{E}_{\mathcal{F}}\left[1-\frac{\pi^{2}}{12}\left(\frac{k T}{\mathcal{E}_{\mathcal{F}}}\right)^{2}\right]
$$

Using (44), (45), an asymptotic expansion for $f_{\frac{5}{2}}$ and (49) we obtain

$$
\frac{\left\langle E^{(Q)}\right\rangle}{\left\langle N^{(Q)}\right\rangle} \simeq \frac{3}{5} \mathcal{E}_{\mathcal{F}}\left[1+\frac{5 \pi^{2}}{12}\left(\frac{k T}{\mathcal{E}_{\mathcal{F}}}\right)^{2}\right]
$$

From (9) and (50) we get an expression for the specific heat in the limit of low temperature

$$
\frac{C_{V}}{\left\langle N^{(Q)}\right\rangle k} \simeq \frac{\pi^{2}}{2} \frac{k T}{\mathcal{E}_{\mathcal{F}}} \simeq \frac{\pi^{2}}{2} \frac{k T}{\mathcal{E}_{\mathcal{F}}{ }^{(0)}}\left(1-\frac{1}{3} \operatorname{Tr}(F)\right)
$$

Writing the entropy using (5) gives the perturbed entropy in the low temperature limit as

$$
S^{(Q)} \simeq C_{V}
$$

as expected from the integrated equation for specific heat. The low-temperature ideal gas law perturbation is

$$
P \simeq \frac{2}{5} n^{(Q)} \mathcal{E}_{\mathcal{F}}\left[1+\frac{5 \pi^{2}}{12}\left(\frac{k T}{\mathcal{E}_{\mathcal{F}}}\right)^{2}\right]
$$

The expectation value for the spin can be calculated in the quantum regime using the fractional occupancies

$$
f(\mathbf{n}, s)=\frac{1}{e^{\alpha} e^{\beta E_{\mathbf{n}, s}}+1},
$$

where $E_{\mathbf{n}, s}=E_{\mathbf{n}, s}^{(0)}+\delta E_{\mathbf{n}, s}$ and the perturbation is as given in (23). With notation as above, the expectation of the spin is given by

$$
\begin{aligned}
\left\langle\mathbf{s}^{(Q)}\right\rangle & =\sum_{\mathbf{n}, s} s \frac{\mathcal{G}(\mathbf{n})}{|\mathcal{G}(\mathbf{n})|} f(\mathbf{n}, s) \\
& \simeq-2 \beta \frac{\pi^{2} \hbar^{2}}{2 m L^{2}} \sum_{\mathbf{n}} \mathcal{G}(\mathbf{n}) \frac{e^{\alpha} e^{\beta \frac{\pi^{2} \hbar^{2}}{2 m L^{2}} n^{2}}}{\left(1+e^{\alpha} e^{\beta \frac{\pi^{2} \hbar^{2}}{2 m L^{2}} n^{2}}\right)^{2}} \\
& \simeq-\left\langle N^{(Q)}\right\rangle\left[2 \frac{\beta}{\lambda^{3}} f_{\frac{1}{2}}\left(e^{-\alpha}\right) \mathbf{B}+\frac{1}{2} \operatorname{Tr}(\mathbf{G})\right]
\end{aligned}
$$

This calculation demonstrates the surprising fact that the contribution of the $G$ term is temperature independent and does not randomize at high temperature. At low temperatures, the contribution from the $B$ term can be written as

$$
\left\langle\mathbf{s}_{B}^{(Q)}\right\rangle \simeq-\left\langle N^{(Q)}\right\rangle \frac{3}{2} \frac{\mathbf{B}}{\mathcal{E}_{\mathcal{F}}}\left[1-\frac{\pi^{2}}{12}\left(\frac{k T}{\mathcal{E}_{\mathcal{F}}}\right)^{2}\right] .
$$

This is identical to the result for a quantum Fermi gas in an external magnetic field when only spin interactions are relevant. Note that the parameter $\mathbf{B}$ is a fixed background vector that does not rotate with the experiment.

\section{QUANTUM GAS - BOSONS}

It is possible to generate a model for a free spin-0 boson gas by combining two fermions into a singlet representation of the spin group. This means that the expectation value of all spin-couplings vanish in the hamiltonian (13). The resulting hamiltonian is given by

$$
H=\frac{p^{2}}{2 m}+A+C_{j} \frac{p_{j}}{m}+F_{j k} \frac{p_{j} p_{k}}{2 m}
$$

Choosing the ground state energy to be zero and employing the notation of the previous sections, the associated grand partition function for the unperturbed case is

$$
\begin{aligned}
\ln \left(Z_{0}^{(Q B)}\left(\alpha_{0}\right)\right)= & -\sum_{\mathbf{n}} \ln \left(1-e^{-\alpha_{0}} e^{-\beta E_{\mathbf{n}}^{(0)}}\right) \\
& -\ln \left(1-e^{-\alpha_{0}}\right)
\end{aligned}
$$

where $E_{\mathbf{n}}^{(0)}=\left(\pi^{2} \hbar^{2} / 2 m L^{2}\right) n^{2}$ as before, and the ground state has been separated out to allow for Bose-Einstein condensation at low temperatures. Approximating the sum as an integral gives the standard result

$$
\ln \left(Z^{(Q B)}\left(\alpha_{0}\right)\right)=\frac{1}{\lambda^{3}} V g_{\frac{5}{2}}\left(e^{-\alpha_{0}}\right)-\ln \left(1-e^{-\alpha_{0}}\right)
$$


where $\lambda$ is the thermal wavelength and $g_{\frac{5}{2}}\left(e^{-\alpha}\right)$ is the Bose-Einstein integral

$$
g_{\nu}\left(e^{-\alpha}\right)=\frac{1}{\Gamma(\nu)} \int_{0}^{\infty} \frac{x^{\nu-1}}{e^{\alpha} e^{x}-1} d x .
$$

Using (3) we obtain the expected number of particles in the excited states and the associated energy as

$$
\begin{aligned}
\left\langle N_{0}^{(Q B)}\left(\alpha_{0}\right)\right\rangle-\left\langle N_{G 0}\right\rangle & =\frac{1}{\lambda^{3}} V g_{\frac{3}{2}}\left(e^{-\alpha_{0}}\right), \\
\left\langle E_{0}^{(Q)}\left(\alpha_{0}\right)\right\rangle & =\frac{3}{2} k T \frac{V}{\lambda^{3}} g_{\frac{5}{2}}\left(e^{-\alpha_{0}}\right),
\end{aligned}
$$

where $\left\langle N_{G 0}\right\rangle=\left[e^{\alpha_{0}}-1\right]^{-1}$ is the expected number of particles condensed into the ground state.

The only nontrivial leading order perturbation in (57) arises from the $F$ term. A calculation which follows that done for the case of fermions gives

$$
\begin{aligned}
\ln \left(Z^{(Q B)}(\alpha)\right) \simeq & \left(1-\frac{1}{2} \operatorname{Tr}(F)\right) \frac{1}{\lambda^{3}} V g_{\frac{5}{2}}\left(e^{-\alpha}\right) \\
& -\ln \left(1-e^{-\alpha}\right) .
\end{aligned}
$$

It follows from (3) and (40)-(43) that for the perturbed case we have

$$
\begin{aligned}
\left\langle N^{(Q B)}(\alpha)\right\rangle-\left\langle N_{G 0}\right\rangle & =\left(1-\frac{1}{2} \operatorname{Tr}(F)\right) \frac{1}{\lambda^{3}} g_{\frac{3}{2}}\left(e^{-\alpha}\right), \\
\left\langle E^{(Q B)}(\alpha)\right\rangle & =\frac{3}{2} k T \frac{V}{\lambda^{3}} g_{\frac{5}{2}}\left(e^{-\alpha}\right) .
\end{aligned}
$$

As in the Fermi case, the chemical potential can be expressed as a function of the number of particles in excited states. The computations, modulo obvious modifications, are similar to (47)-(53). Because only $\operatorname{Tr}(F)$ enters into the grand partition function, it is possible to use the concept of effective mass to absorb the effect as before. Standard results of Bose-Einstein condensation therefore hold in a given laboratory frame. The effect is nontrivial, as in the fermion case, because boosting the experiment will change the effective mass. Modifications of these calculations are required if the ground state wave function does not exhibit rotational symmetry, such as is the case in actual condensate experiments due to some optical and magnetic trapping configurations 34. In this case, the zero point of energy can depend on orientation of the apparatus and induce rotational variations in the condensate properties. An analysis of this type for specific experiments would be interesting, but is beyond the scope of the paper.

\section{CONCLUSION}

The formalism of statistical mechanics in the presence of symmetry violation parallels the conventional situation. The laws of thermodynamics are the same as in the conventional case, although the specific expectation values of thermodynamic quantities can be modified by the Lorentz-violating terms. Our approach involves an assumption of a maximal lack of information (or entropy) regarding a system subject to various constraints imposed by the physical observables. The temperature and chemical potentials can be defined simply as the lagrange multipliers associated with the constraints, hence two systems in equilibrium automatically have the same temperature and chemical potential as there is only one lagrange multiplier for each overall constraint. This method in fact produces equivalent results to the more conventional assumption of equal a-priori statistics, however, it has the advantage of providing straightforward definitions of thermodynamic variables with conventional equilibrium properties.

As an explicit example, the unperturbed system was assumed to be an ideal gas in the absence of any external applied fields (such as magnetic or gravitational fields). Expectation values for scalar thermodynamic quantities such as energy and particle number were unaltered except for an overall scaling factor $\operatorname{Tr}(F)$. This happens because the unperturbed system is rotationally invariant and scalar expectation values can only be corrected by perturbations with commensurate symmetry. This can also be incorporated as an effective mass $m^{*}=\left(1-\frac{1}{3} \operatorname{Tr}(F)\right) m$ in the hamiltonian, although the effective mass defined in this way depends on the observer's Lorentz frame. For instance, a gas in motion on the surface of the Earth would exhibit slight sidereal variations in effective mass due to changes in $\operatorname{Tr}(F)$. This effect is relativistically suppressed and unlikely to be observable for physically reasonable values for the violation parameters.

More interesting is the net spin expectation value contributed by the terms that couple to the spin. The purespin coupling $B_{j}$ mimics a constant background magnetic field and induces a corresponding magnetic moment per unit volume in the gas. Any additional applied magnetic field could be added to this term to calculate the net result on an actual experiment. For example, an Earthbased experiment will rotate in space and the constant background vector will interfere with the applied magnetic field to produce a sidereal variation.

The derivative-spin coupling $G_{i j k}$ generates a fundamentally new type of effect that induces a temperatureindependent polarization in the classical gas that is proportional to $\operatorname{Tr}(\mathbf{G})$. This means that even at very high temperatures there will be a net residual polarization that does not randomize. This is a reasonable result, considering that the $\operatorname{Tr}(\mathbf{G})$ term couples the spin to the conventional kinetic energy term in the hamiltonian and indicates that any physical effects should scale accordingly. An effect of this type should be clearly distinguishable from stray magnetic field effects that have an inverse temperature dependence.

In the zero temperature limit, both the $B_{j}$ and $G_{i j k}$ terms contribute a polarization to the Fermi gas. The $\mathbf{B}$ contribution depends on $n^{1 / 3}$ while the $\operatorname{Tr}(\mathbf{G})$ depends linearly on particle density $n$. This means that the effects of the $\operatorname{Tr}(\mathbf{G})$ term can grow significantly faster with increasing density than conventional magnetic field effects. 
[1] V.A. Kostelecký and S. Samuel, Phys. Rev. D 39, 683 (1989); ibid. 40, 1886 (1989); Phys. Rev. Lett. 63, 224 (1989); ibid. 66, 1811 (1991); V.A. Kostelecký and R. Potting, Nucl. Phys. B 359, 545 (1991); Phys. Lett. B 381, 89 (1996); Phys. Rev. D 63, 046007 (2001); V.A. Kostelecký, M. Perry, and R. Potting, Phys. Rev. Lett. 84, 4541 (2000).

[2] D. Colladay and V.A. Kostelecký, Phys. Rev. D 55, 6760 (1997); Phys. Rev. D 58, 116002 (1998).

[3] D. Colladay and P. McDonald, J. Math. Phys. 433554 (2002).

[4] For a summary of recent theoretical models and experimental tests see, for example, CPT and Lorentz Symmetry, V.A. Kostelecký, ed., World Scientific, Singapore, 1999; CPT and Lorentz Symmetry II, V.A. Kostelecký, ed., World Scientific, Singapore, 2002.

[5] S. M. Carroll, et al., Phys. Rev. Lett. 87141601 (2001); Z. Guralnik, et al., Phys. Lett. B 517450 (2001).

[6] C. D. Froggatt and H. B. Nielsen, hep-ph/0211106

[7] V. A. Kostelecký and R. Lehnert, Phys. Rev. D 63, 065008 (2001).

[8] KTeV Collaboration, H. Nguyen, in Ref. [4]; OPAL Collaboration, R. Ackerstaff et al., Z. Phys. C 76, 401 (1997); DELPHI Collaboration, M. Feindt et al., preprint DELPHI 97-98 CONF 80 (1997); BELLE Collaboration, K. Abe et al., Phys. Rev. Lett. 86, 3228 (2001); BaBar Collaboration, B. Aubert et al., hep-ex/0303043 FOCUS Collaboration, J.M. Link et al., Phys. Lett. B 556, 7 (2003).

[9] D. Colladay and V.A. Kostelecký, Phys. Lett. B 344, 259 (1995); Phys. Rev. D 52, 6224 (1995); Phys. Lett. B 511, 209 (2001); V.A. Kostelecký and R. Van Kooten, Phys. Rev. D 54, 5585 (1996); O. Bertolami et al., Phys. Lett. B 395, 178 (1997); N. Isgur et al., Phys. Lett. B 515, 333 (2001).

[10] V.A. Kostelecký, Phys. Rev. Lett. 80, 1818 (1998); Phys. Rev. D 61, 016002 (2000); Phys. Rev. D 64, 076001 (2001).

[11] L.R. Hunter et al., in V.A. Kostelecký, ed., CPT and Lorentz Symmetry, World Scientific, Singapore, 1999; D. Bear et al., Phys. Rev. Lett. 85, 5038 (2000); D.F. Phillips et al., Phys. Rev. D 63, 111101 (2001); M.A. Humphrey et al., Phys. Rev. A 68, 063807 (2003); Phys. Rev. A 62, 063405 (2000); V.A. Kostelecký and C.D. Lane, Phys. Rev. D 60, 116010 (1999).

[12] R. Bluhm et al., Phys. Rev. Lett. 88, 090801 (2002); Phys. Rev. D 68, 125008 (2003).

[13] F. Canè et al., physics/0309070

[14] H. Dehmelt et al., Phys. Rev. Lett. 83, 4694 (1999); R. Mittleman et al., Phys. Rev. Lett. 83, 2116 (1999); G. Gabrielse et al., Phys. Rev. Lett. 82, 3198 (1999); R. Bluhm et al., Phys. Rev. Lett. 82, 2254 (1999); Phys. Rev. Lett. 79, 1432 (1997); Phys. Rev. D 57, 3932 (1998).

[15] B. Heckel, in Ref. 4]; L.-S. Hou, W.-T. Ni, and Y.-C.M. Li, Phys. Rev. Lett. 90, 201101 (2003); R. Bluhm and V.A. Kostelecký, Phys. Rev. Lett. 84, 1381 (2000).

[16] H. Müller, S. Herrmann, A. Saenz, A. Peters, and C. Lämmerzahl, Phys. Rev. D 68, 116006 (2003).
[17] S.M. Carroll, G.B. Field, and R. Jackiw, Phys. Rev. D 41, 1231 (1990); M.P. Haugan and T.F. Kauffmann, Phys. Rev. D 52, 3168 (1995); V.A. Kostelecký and M. Mewes, Phys. Rev. Lett. 87, 251304 (2001).

[18] R. Jackiw and V.A. Kostelecký, Phys. Rev. Lett. 82, 3572 (1999); C. Adam and F.R. Klinkhamer, Nucl. Phys. B 657, 214 (2003); H. Müller, C. Braxmaier, S. Herrmann, A. Peters, and C. Lämmerzahl, Phys. Rev. D 67, 056006 (2003); T. Jacobson, S. Liberati, and D. Mattingly, Phys. Rev. D 67, 124011 (2003); V.A. Kostelecký and A.G.M. Pickering, Phys. Rev. Lett. 91, 031801 (2003); R. Lehnert, Phys. Rev. D 68, 085003 (2003); G.M. Shore, Contemp. Phys. 44, 503 2003; B. Altschul, Phys. Rev. D 69, 125009 (2004); hep-th/0407172

[19] V. A. Kostelecký, M. Perry, and R. Lehnert, Phys. Rev. D 68, 123511 (2003).

[20] J. Lipa et al., Phys. Rev. Lett. 90, 060403 (2003); H. Müller et al., Phys. Rev. Lett. 91, 020401 (2003); P. Wolf et al., $\mathrm{gr}-\mathrm{qc} / 0401017$

[21] V.A. Kostelecký and M. Mewes, Phys. Rev. D 66, 056005 (2002); V.A. Kostelecký and Q. Bailey, hep-ph/0407252

[22] V.W. Hughes et al., Phys. Rev. Lett. 87, 111804 (2001); R. Bluhm et al., Phys. Rev. Lett. 84, 1098 (2000).

[23] S. Coleman and S.L. Glashow, Phys. Rev. D 59, 116008 (1999); V. Barger, S. Pakvasa, T.J. Weiler, and K. Whisnant, Phys. Rev. Lett. 85, 5055 (2000); J.N. Bahcall, V. Barger, and D. Marfatia, Phys. Lett. B 534, 114 (2002); V.A. Kostelecký and M. Mewes, hep-ph/0308300 S. Choubey and S.F. King, Phys. Lett. B 586, 353 (2004).

[24] V.A. Kostelecký and M. Mewes, Phys. Rev. D 69, 016005 (2004); hep-ph/0406255

[25] D. Colladay and P. McDonald, J. Math. Phys. 45, 3228 (2004).

[26] M. S. Berger and V.A. Kostelecký, Phys. Rev. D 65, 091701 (2002); M. S. Berger, Phys. Rev. D 68, 115005 (2003).

[27] V. A. Kostelecký, Phys. Rev. D 69, 105009 (2004).

[28] O.Bertolami, et al., Phys. Lett. B 395, 178 (1997).

[29] E. T. Jaynes, Phys. Rev. 106, 620 (1957).

[30] V.A. Kostelecký and C.D. Lane, J. Math. Phys. 40, 6245 (1999).

[31] Note that the components of $\mathbf{p}$ are written as $p_{j}$ in this paper and the nonrelativistic metric is taken to be $\delta_{j k}$. These components correspond to the components $p^{j}$ defined in reference [30]. This accounts for the sign changes that occurr in the odd momentum terms in the hamiltonian.

[32] The $A$ term can make important contributions to the physics in the relativistic limit where energy splittings between particles and antiparticles become relevant, as is the case in the baryogenesis calculations performed in reference [28].

[33] R. K. Pathria, Statistical Mechanics, Oxford Press, Boston, 1996.

[34] For a review of Bose-Einstein condensation see, for example, F. Dalfovo, et al., Rev. Mod. Phys. 71, 463 (1999). 